\title{
Clinical Applications of Stochastic Dynamic Models of the Brain, Part II:
}

A Review

\author{
James A Roberts ${ }^{1,2}$, Karl J. Friston ${ }^{3}$, Michael Breakspear ${ }^{1,4}$
}

${ }^{1}$ Systems Neuroscience Group, QIMR Berghofer Medical Research Institute, Herston, Brisbane, QLD 4006, Australia

${ }^{2}$ Centre for Integrative Brain Function, QIMR Berghofer Medical Research Institute, Herston, Brisbane, QLD 4006, Australia

${ }^{3}$ The Wellcome Trust Centre for Neuroimaging, Institute of Neurology, University College London, London, UK

${ }^{4}$ Metro North Mental Health Service, Royal Brisbane and Women's Hospital, Brisbane, QLD, Australia

* Corresponding author: Michael Breakspear, Systems Neuroscience Group, QIMR Berghofer Medical Research Institute, 300 Herston Rd, Herston, QLD 4006, Australia. Email: michael.breakspear@qimrberghofer.edu.au, phone: +61 73845 3692, fax: +61 733620111.

Keywords: stochastic, mathematical modeling, epilepsy, melancholia, computational psychiatry

Word counts:

Abstract: 152

Article body: 3996

Number of figures: 3

Number of tables: 0

Number of supplemental items: 0

Short title: Stochastic Dynamic Models in Psychiatry and Neurology 


\begin{abstract}
:
Brain activity derives from intrinsic dynamics (due to neurophysiology and anatomical connectivity) in concert with stochastic effects that arise from sensory fluctuations, brainstem discharges and random microscopic states such as thermal noise. The dynamic evolution of systems composed of both dynamic and random fluctuations can be studied with stochastic dynamic models (SDMs). This paper, Part II of a two-part series, reviews applications of SDMs to large-scale neural systems in health and disease. Stochastic models have already elucidated a number of pathophysiological phenomena; such as epilepsy and hypoxic ischemic encephalopathy, although their use in biological psychiatry remains rather nascent. Emerging research in this field includes phenomenological models of mood fluctuations in bipolar disorder and biophysical models of functional imaging data in psychotic and affective disorders. Together with deeper theoretical considerations, this work suggests that SDMs will play a unique and influential role in computational psychiatry, unifying empirical observations with models of perception and behavior.
\end{abstract}




\section{Clinical applications of SDMs}

Brain activity arises from an interplay of nonlinear dynamics with stochastic fluctuations. In Part I of this two-part series, we provided a primer on stochastic dynamical models (SDMs) equations that govern the behavior of such systems (1). Here, in Part II, we review how SDMs have furnished insights into clinical problems: We begin with a survey of neurological disorders, where there exists a rich history of stochastic neural modeling. We then consider applications to psychiatric disorders, a less explored avenue. We will also consider sleep and, eye movements and decision making, which are commonly disturbed in psychiatric disorders.

\subsection{Epilepsy}

Mathematical models of epileptic seizures are numerous, using a wide range of methods, and spanning small and large scales (2). Here, we focus on research that has treated the stochastic aspects of seizures. A major role for noise in models of seizures is to explain their abrupt and unexpected onset. One plausible explanation for sudden seizure onset comes from the interplay of stochastic perturbations with the underlying dynamical structure. The key ingredient is bistability between non-seizure and seizure states (3-7). This means that for the same parameter values, the model can be in one of two states (seizure vs. non-seizure). In this scenario, seizure onset arises when noisy perturbations "kick" the brain onto the pathological seizure attractor (Fig. 1B). In spatially-extended models, this bistability can also explain the spread of seizures into neighboring cortical regions (8).

The bistable mechanism has been shown to reproduce distributions of ictal and interictal durations in both humans and animal models (9). Indeed bistable switching is applicable to many phenomena (10). Other interactions between noise and the dynamics of seizures offer important insights into the possible bifurcation structures that explain specific seizure phenomena in electrophysiological recordings $(5,11)$. Similarly, interplay between stochastic and deterministic

dynamics affects seizure durations in coupled neuronal networks (12). A common thread to these 
studies is the analysis of distributions of times spent in the different states or spectral signatures. Analysis of these higher-order statistics provides a stronger test of models than simple summary statistics alone, and is a powerful approach in studies of stochastic models more generally.

According to an alternative proposal, seizure onset corresponds to a bifurcation from a fixedpoint attractor onto a limit cycle under the influence of a slowly changing physiological parameter (e.g. 'excitability'), with seizure termination corresponding to a similar transition in the opposite direction $(2-5,11,13,14)$. The physiological consequences of the ensuing energydepleting large amplitude oscillations lead to opposing (homeostatic) physiological changes that restore stability of the fixed point. While noise plays a relatively subordinate role in triggering 'bifurcation seizures', stochastic fluctuations nonetheless become evident just before seizure onset - at the cusp of the bifurcation - exhibiting critical slowing, as observed in human and non-human, invasive and noninvasive data $(9,11,15-18)$. This slowing offers a candidate method for seizure prediction. However, this goal remains elusive, despite promising preliminary results (19), speaking to a need for more high-quality data (20).

Both bistability and bifurcations have been invoked for a range of generalized and localized seizures: Despite considerable work, there remains a lack of consensus (for example absence seizures have been proposed as arising either through bistability (21) or a bifurcation (3)). Future work should aim to reconcile these possibilities.

\subsection{Parkinson's disease}

Stochastic models of coupled neurons (22) and mean-field population activity (23) have been used to explore the impact of reduced dopamine (a hallmark of Parkinson's disease) on the dynamics of feedback loops linking cortex, thalamus, and basal ganglia. These noise-driven models explain the emergence of oscillations, synchrony, and other spectral changes observed in Parkinsonian patients. Other studies using coupled-neuron models have explored the cognitive effects of reduced dopamine in the basal ganglia on working memory (24) and learning (25), yielding predictions that have been verified experimentally (26). 
Stochastic models have also yielded insights into deep-brain stimulation (DBS) protocols for controlling Parkinsonian tremors . Models of coupled basal ganglia and thalamic neurons explain the ability of high-frequency DBS to suppress pathological oscillations $(27,28)$. Computational approaches also provide a suitable test bed for optimizing stimulation protocols (29, 30). Systematic analysis of a spiking-neuron model has suggested novel stochastic stimulation protocols offer similar therapeutic benefits to high-frequency DBS but for lower current amplitudes (31), although this remains to be tested. Methods for extinguishing oscillations have been tested in animal and slice models $(32,33)$, and also for closed-loop control paradigms in macaque (34) and human (35). Although evidence from large clinical trials has yet to emerge, there is optimism that novel stimulation paradigms will improve on existing DBS protocols, at the very least because manual adjustment is difficult, and even modest decreases in energy use could improve battery life in implanted devices (29). Stochastic phase-oscillator models phenomenological models used to describe oscillatory dynamics where the state variables are phases and (possibly) amplitudes - underpin the method of stochastic phase resetting (36), a well-studied protocol for Parkinson's disease (37). Phase resetting refers to the precise delivery of stimuli to alter the phase of an oscillator with the aim of changing its degree of synchronization with other oscillators. These methods have also found application to other disorders involving abnormal synchrony, including obsessive-compulsive disorder (OCD) (38) and tinnitus (39).

\subsection{Burst suppression}

Across a range of healthy and clinical settings, cortical activity exhibits burst suppression highly-erratic bursts punctuating an otherwise low-amplitude background (40). Neonatal EEG exhibits pronounced burst suppression during recovery from hypoxia at birth. Recently it was shown that the bursts in post-hypoxic neonatal EEG exhibit scale-free dynamics (41), a hallmark of stochastic systems near a critical point characterized by fractal behavior with no preferred spatial or temporal scales (42). Using the tools of statistical physics to quantify these bursts opens the prospect of predicting clinical outcomes (43). The potential utility of these EEG metrics is underwritten by the fact that they also have diagnostic (44) and prognostic (45) utility when applied to bursts in preterm EEG. A leading candidate stochastic theory for burst 
suppression rests on an interaction between noisy brain activity and metabolism (46, 47). Evidence for this comes from detailed modeling of coupled neurons (46), and from phenomenological stochastic modeling (41). Although the former coupled-neuron model includes noise, it is not central to the mechanism for the limit-cycle bursts (rather, it improves the biological realism of the neuronal activity). In contrast, for the latter (phenomenological) model, stochastic dynamics are central to the bursting mechanism: a key statistical signature of the bursts (an asymmetric burst shape) was found to emerge from a stochastic model with statedependent slow-timescale dynamics, a proxy for metabolic depletion (41).

\subsection{Anesthesia}

Loss of consciousness in anesthesia is accompanied by marked changes in EEG, which are routinely used to monitor the depth of sedation. Stochastic neural mean-field modeling has explained these EEG spectral changes as signatures of a phase-transition at the boundary between conscious and comatose states (48). Here, the model's stochastic nature is crucial to explain the clinically-observed peaks in EEG power at the transitions. Noise-driven mean-field models have also been used to explore other anesthetic-induced changes to EEG spectra (49-51). Models have also recently been applied in state-estimation methods for automated tracking of brain states during anesthesia (52).

\subsection{Bipolar disorder}

Fluctuating disturbances in mood are the hallmark of bipolar disorder. There is conflicting evidence from time-series analysis methods as to whether these dynamics are predominantly nonlinear oscillations (53) or linear noise (54). Coupled relaxation oscillators with noise have been used as a phenomenological model of mood (55). In this model, noise drives instability in mood, and patient-specific noise levels can be inferred from data. Other deterministic limit-cycle models of mood could likely be extended to the stochastic domain (56). Another phenomenological model focused on an abstract measure of behavioral activation rather than mood (57), showing that increased nonlinearity in the oscillatory component of mood fluctuations drives switching between multistable high- and low-activity states. Analysis of 
dwelling time scales may thus prove a fruitful test of competing models (10). Mood oscillations in bipolar disorder have also been modeled with dynamics typical of biochemical reactions (58, 59), hinting at more biophysically-motivated mechanisms.

The analysis of mood dynamics offers potential clinical applications beyond increased understanding of the pathophysiology: detailed tracking of mood together with time-series analysis models may yield novel biomarkers of mood instability (60). Extending this approach to SDMs of the underlying neural activity (as opposed to simply the time-series data) may make any putative biomarkers more robust. However such advances require a more principled understanding of the neurobiological nature of mood, affect, and their relationship to brain networks integrating autonomic, introspective, and cognitive processes (61-63).

\subsection{Melancholia}

While there is a considerable body of computational research into the cognitive biases that accompany depression (64), little of this has been informed by SDMs. As in bipolar disorder, mood during depressive episodes fluctuates considerably, albeit without the manic highs of bipolar disorder. Self-reported mood ratings scored intermittently throughout the day evidence increased correlation and variance prior to the onset of clinical depressive episodes in those with a history of depression (65). These symptom fluctuations are consistent with critical slowing in a noise-driven system near a bifurcation, such as exemplified in a proof-of-principle Langevin equation modeling mood directly as a state variable (65).

What are the neuronal correlates of these mood fluctuations? Two recent studies $(66,67)$ have modeled stochastic neural dynamics in patients with melancholia, a putative subtype of depression characterized by neurovegetative symptoms such as psychomotor slowing, anhedonia, and diurnal mood variation (68). Functional neuroimaging data across multiple brain regions was studied using stochastic Dynamic Causal Modeling (sDCM, (69)), which models the neuronal activity in each region $x_{i}$ as

$$
\frac{d x_{i}}{d t}=\mathrm{A}^{(i)} \mathbf{x}+\mu_{i} \eta_{i}
$$


where $\mathrm{A}^{(i)}$ is the $i$-th row of the matrix $\mathbf{A}$ that describes coupling - or effective connectivity between the states. In this simple Langevin equation, noisy inputs $\eta_{i}$ drive local fluctuations which spread to other regions through the effective coupling matrix A (Figure 2). A forward model predicts BOLD fluctuations, allowing inversion of the SDCM from empirical fMRI data and estimation of $\mathbf{A}$.

Patients with melancholia report a distinct and intrusive dysphoric state during internallygenerated thought. Application of sDCM to resting-state fMRI (66) was used to study the correlates of these self-reports, comparing patients with melancholia to healthy controls and those with non-melancholic depression. Melancholia was associated with weaker influences among a network of cortical regions, particularly the influence of the anterior insula on frontoparietal and anterior cingulate nodes. It was hence inferred that the impoverished quality of internally-generated thought in melancholia reflects a diminished influence of interoceptive fluctuations on attention and cognitive control.

Self-reported mood likely reflects a combination of "internal" mood states and subjective responses to emotionally-salient experiences. Stochastic DCM has also been applied to fMRI data acquired while melancholic participants viewed sad and funny movie clips (67). In this setting, the stochastic term in Equation (1) is therefore a mixture of endogenous fluctuations and sensory perturbations from the movies. Melancholia was associated with network-wide increases in effective connectivity, particularly between the attention (fronto-parietal) and interoceptive (anterior insula) regions, when shifting from rest to negative film viewing. This was suggested to reflect disrupted attentional resource allocation, particularly in switching between interoceptive and exteroceptive signals.

These observations are consistent with the speculative proposal that illness states, such as melancholia, are themselves dynamic attractors that unfold on slow time scales, and that fluctuations (such as stressors) trigger noise-driven switches between euthymia and affective disturbances. 


\subsection{Sleep}

A neural mass model has also been developed for the brainstem nuclei controlling sleep-wake transitions (70). In this model, as in sleep, most time is spent in either wake or sleep states, with relatively rapid transitions in between. The circadian rhythm slowly drives the model alternately between wake and sleep. In between these states is a bistable region. Much like the epilepsy example above, noisy perturbations can trigger erratic transitions between the two states (71). This provides an explanation for the sleep-wake patterns in narcolepsy, which is characterized by difficulty in staying awake, and (perhaps paradoxically) also difficulty in remaining asleep. For choices of model parameters in this bistable region between wake and sleep, noisy stimuli are sufficient to yield fragmented sleep patterns in excellent agreement with clinical narcolepsy phenotypes (Fig. 3).

A further role for noise in sleep modeling is characterizing the changes in dynamics observed near a transition. As in the examples above, the dynamical approach to a bifurcation yields quantifiable changes in the dynamics - critical slowing - that indicate an imminent transition. For sleep transitions, the sleep model reproduces power spectral changes at sleep onset (72), potentially useful in sleep-monitoring applications (73). It is also possible to automatically track arousal states by inferring the parameters of a biophysical model fitted to EEG data $(74,75)$. This highlights one of the benefits of using models to provide an objective, automated alternative to traditional clinician-determined ratings.

\subsection{Working memory and decision making}

While less visible than the classic psychotic symptoms of delusions and hallucinations, cognitive impairments in schizophrenia nonetheless constitute much of the illness burden. A rich body of work posits cognitive states, such as working memory, as fixed point attractors in a multistable dynamic landscape (76-80). Cortical network models then pinpoint the role of specific neurobiological processes underlying the corresponding persistent activity, particularly the role of recurrent excitatory (NMDA) receptors (81). Disturbances in NMDA-mediated feedback have been proposed to decrease the depth of the basin of attraction surrounding the working memory 
attractor (82). Stochastic neuronal fluctuations then render the working memory attractor unstable, yielding shorter dwell times corresponding to impaired working memory.

Models of working memory have also explored the role of dopamine in prefrontal cortex (83), and its role in cognitive disturbances in schizophrenia $(84,85)$. In this theory, multistability is again crucial. Different attractors represent different states, and modulation (via dopamine) of the "height" of the barriers between the attractors determines the ease with which states switch. Positive symptoms of schizophrenia are then associated with low barriers and overly-frequent switching, while negative symptoms correspond to high barriers and excessive inflexibility. A noise-driven mesoscopic rate model of cortical activity has also been employed to explain the decrease in the global mean signal observed in resting state fMRI in schizophrenia (86). Further applications of modeling approaches to neuroimaging and mechanisms of schizophrenia have been reviewed recently $(87,88)$.

Decision making is inherently noisy and thus a prime candidate for SDMs. The most widely used phenomenological model for two-choice decision making is the "drift-diffusion model", describing random accumulation of information in favor of competing choices $(89,90)$. Driftdiffusion models have been applied to schizophrenia (91), attention deficit hyperactivity disorder (92), and OCD (93). Many of these analyses employ model inversion, similar to the DCM studies reviewed above, but where the fitted dynamic model describes the abstract evidenceaccumulation leading to a decision.

Neurobiological models of decision making posit that cognitive states are encoded by stochastic attractors in the corresponding cortical regions. Noise-driven recurrent cortical network models (94, 95) have described the neuronal attractors underlying the encoding of accumulating evidence and the corresponding bifurcation at the point of decision $(96,97)$. Such models reproduce salient characteristics of decision-correlated neural activity in parietal and prefrontal cortex as well as the decision dynamics (accuracy and reaction times). The central role of stochastic effects in decision making is underlined by quantitative analyses of behavioral variability (principally in the trial-to-trial reaction time) during adaptive behavior (98), its 
evolution during development $(99,100)$, healthy ageing (101), and recovery from brain injury (102), as well as its relationship to BOLD signal variability (103-105).

Modeling basins of attraction have also found application in models of OCD. Symptoms of OCD include an inflexibility, associated with being "trapped" in a brain state, associated with increased attractor basin depth (106-108). Using a stochastic neurocomputational model incorporating the modulatory role of serotonin on orbitofrontal function, it has been shown that simulated low serotonin leads to the model getting trapped in specific attractor states ("perseverative" activity) and an increased tendency to form strong attractors (a worsening of existing obsessions) (109).

\subsection{Eye movements}

Abnormal eye movements occur in various disorders across the lifespan, including autism, schizophrenia, Parkinson's and Alzheimer's disease. Models of eye movements in schizophrenia have been reviewed recently (110): We briefly draw attention to the role of stochastic dynamics in this field.

Eye movements are easily measured and manipulated in experiments, while still being closely tied to many higher-order cognitive processes that are disturbed in patient populations. In stochastic models of eye movement, noise is often used to represent uncertainty, whether in sensory inputs, motor outputs, or perception. For example, a widely-employed task requires participants to make saccadic eye movements away from visual stimuli that appear randomly in one of four peripheral locations. Patients with schizophrenia are more variable (and error-prone) in this task. This has been modeled using a network of coupled neurons with noisy inputs (111). Differences in eye movement variability between patients and controls were captured by group differences in the input noise levels. This work suggests that the increased variability arises specifically in the noisy accumulation of information rather than other hypothesized processes such as differences in the threshold for deciding to perform a saccade. 
Another classic eye movement task is smooth pursuit, whereby participants track a moving target with their eyes. Brief visual occlusions can be used to introduce uncertainties in future target positions (112). Schizophrenia is associated with small errors during smooth pursuit plus overshoot errors following occlusion. This uncertainty in target position has been modeled within the framework of "active inference" (113), where action and perception are modeled hierarchically, specifically encoding uncertainty in states. This model of smooth pursuit behavior explains suboptimal tracking performance in schizophrenia $(112,114)$, and has recently been extended to model corresponding neuronal activity (115). Schizophrenic patients also have

difficulty fixating on non-moving targets. Fixation stability during free viewing was the single best predictor of schizophrenia in a recent classifier (116), and there exist models of fixational eye movements $(117,118)$. Thus, as noted recently (110), models of fixational eye movements have potential for future application to schizophrenia.

\section{Future perspectives}

The fusion of dynamical systems theory with statistical physics provides a powerful and unifying framework for modeling clinical disorders of the brain. Noise interacts with the underlying dynamic flow to yield a variety of adaptive neural behaviors, which include slow and fast fluctuations, multistable switching, and simple attracting dynamics. Subtle disturbances in the balance of noise and order yields maladaptive dynamics such as trapping, unstable fluctuations, and the erratic appearance of abnormal waveforms.

\subsection{General observations}

Several common themes emerge from the above examples, which summarize the main roles for stochastic dynamics in models of clinical disorders:

(i) Stochastic models often treat inputs from external populations or the environment as random fluctuations. Most of the applications reviewed involve this use of noise. However, noise can also be seen as capturing complex dynamics that are not explicitly modeled. This 
underlines the importance of allowing the noise to be imbued with structure, such as statedependence or correlations.

(ii) Measurement noise: A major application of modeling is to infer model parameters from neuroimaging data (i.e., model inversion), enabling estimation of otherwise inaccessible brain states. Model inversion schemes and state estimation techniques must separate desired biological signals, including stochasticity in the generative process, from contaminating measurement noise: these must be modeled separately (119).

(iii) Noise as a driver of spontaneous transitions: In a multistable system, noise can kick the system between states (Fig. 1). This general mechanism explains spontaneous transitions that occur in epilepsy, narcolepsy, and the alpha rhythm. Noise is also crucial in decision making, knocking the system from the working memory ("mnemonic") attractor into one of the several competing decision attractors. However, multiplicative noise can lead to trapping, where the system dwells longer in each state than expected by chance. Multistability comes in different flavours depending upon the nature of the underlying bifurcations $(120,121)$ and the interplay of noise and dynamics (10). Careful disambiguation of these in empirical data may thus elucidate specific mechanisms of multistability in different disorders, and a taxonomy of its adaptive roles.

(iv) Noise as a dynamical signature: Interplay between noise and an impending change of state (i.e., a bifurcation) is a general phenomenon, with particular dynamical signatures giving clues as to the type of bifurcation (15). Critical slowing has been invoked to explain mechanisms related to seizure onset and termination, melancholic mood fluctuations, anesthesia, and sleep-wake transitions.

(v) Fitting to distributions rather than comparing simple summary statistics: On a more practical note, bistable switching and noise signatures of nearby bifurcations are both examples where it is fruitful to analyze statistical distributions derived from data and compare these to the statistics of model dynamics. This is distinct from state estimation methods that act directly on the time series. Such higher-order statistical agreement is a sharp test for putative mechanisms.

(vi) Noise representing uncertainty: At the cognitive level, randomness is central to much of the variability in human perception and behavior, particularly where these pertain to psychiatric disorders. In theories of perception and action, SDMs explicitly encode variance as a 
measure of 'confidence' in estimates about the world (or indeed oneself) (122). Examples reviewed above include models of decision making, working memory, and eye movements (all of which are disrupted in schizophrenia, for example).

(vii) Role of time scales: Neuronal activity and cognition possess multiple spatial and temporal scales (123). Often, stochastic models simplify the treatment of these scales, such as exploiting time scale separation by slaving fast processes to slow ones, thus reducing model complexity. On other occasions, stochastic models shed light on emergent time scales in important clinical phenomena of a dynamic nature, such as sleep-wake transitions, the erratic bursts of seizures and burst suppression, and unstable mood fluctuations in bipolar disorder. Other temporal phenomena, such as the acceleration of speech and thinking in mania, have not yet been addressed.

Clinical applications to psychiatric disorders are promising, albeit less developed than neurological disorders. Psychiatric disorders are characterized by fluctuating severity in key symptom domains, associated with emerging evidence of accompanying changes in neuronal dynamics. Stochastic modeling may assist in redressing the problems currently facing psychiatric nosology. In particular, it has been suggested $(124,125)$ that classification algorithms applied to model parameters may be more accurate and predictive than if they are applied directly to symptom scores or functional imaging time series (64). Applications to major depression suggest that psychiatric disorders may represent self-reinforcing attractors unfolding on slow time scales - heralded by critical slowing then accompanied by their own structural stability. Accordingly, the parameters of the corresponding models could represent nosological latent variables (126). At a time when current symptom-based classification schemes are under increasing scrutiny (127), such fresh approaches are worth pursuing.

While models of brain dynamics remain removed from a precise description of how cognitive phenomena emerge, bridging this gap, if only phenomenologically at first, is likely profitable. One possible area here is in Parkinson's patients undergoing deep brain stimulation to control their tremors. A common side effect is impulsivity (128) - a future direction for models of Parkinsonian brain dynamics could be to include noisy decision-making circuitry. Decision making more broadly offers huge scope for stochastic modeling given the rich variety of possible 
experimental paradigms. One possibility is in the study of mood disorders, which have been linked via formal Bayesian theories to decision making and reward valuation (129). For example, formal models of reward-related learning deficits in depression (130) would be a fruitful target for stochastic dynamic modeling, particularly at the interface with neuroimaging data (122). Another possibility is in the growing areas of cooperative games and neuroeconomics (131). This

field draws on the mathematical areas of game theory and finance. Since dysfunctional social interactions are central to many psychiatric disorders, experiments targeting these social impairments, coupled with appropriate stochastic models, could elucidate the core mechanisms. Stochastic dynamic models will thus be a powerful tool in computational psychiatry.

\section{Acknowledgements}

This work was supported by the National Health and Medical Research Council (Program Grant 1037196), the Australian Research Council (Centre of Excellence for Integrative Brain Function CE140100007), and the Wellcome Trust (Wellcome Trust Principal Research Fellowship: 088130/Z/09/Z).

\section{Financial disclosures}

The authors declare no conflicts of interest and have no financial disclosures. 


\section{References:}

1. Roberts JA, Friston KJ, Breakspear M (2016): Clinical applications of stochastic dynamic models of the brain, Part I: A primer. (submitted).

2. Wendling F, Benquet P, Bartolomei F, Jirsa V (2016): Computational models of epileptiform activity. J Neurosci Methods. 260:233-251.

3. Breakspear M, Roberts JA, Terry JR, Rodrigues S, Mahant N, Robinson PA (2006): A unifying explanation of primary generalized seizures through nonlinear brain modeling and bifurcation analysis. Cereb Cortex. 16:1296-1313.

4. Lopes da Silva F, Blanes W, Kalitzin SN, Parra J, Suffczynski P, Velis DN (2003): Epilepsies as dynamical diseases of brain systems: basic models of the transition between normal and epileptic activity. Epilepsia. 44:72-83.

5. Kramer MA, Truccolo W, Eden UT, Lepage KQ, Hochberg LR, Eskandar EN, et al. (2012): Human seizures self-terminate across spatial scales via a critical transition. Proc Natl Acad Sci U S A. 109:21116-21121.

6. Suffczynski P, Kalitzin S, Lopes Da Silva FH (2004): Dynamics of non-convulsive epileptic phenomena modeled by a bistable neuronal network. Neuroscience. 126:467-484.

7. Benjamin O, Fitzgerald THB, Ashwin P, Tsaneva-Atanasova K, Chowdhury F, Richardson MP, et al. (2012): A phenomenological model of seizure initiation suggests network structure may explain seizure frequency in idiopathic generalised epilepsy. The Journal of Mathematical Neuroscience. 2:1-30.

8. Kim JW, Roberts JA, Robinson PA (2009): Dynamics of epileptic seizures: Evolution, spreading, and suppression. J Theor Biol. 257:527-532.

9. Suffczynski P, Silva FH, Parra J, Velis DN, Bouwman BMG, Van Rijn CM, et al. (2006): Dynamics of epileptic phenomena determined from statistics of ictal transitions. Biomedical Engineering, IEEE Transactions on. 53:524-532.

10. Freyer F, Roberts JA, Ritter P, Breakspear M (2012): A canonical model of multistability and scale-invariance in biological systems. PLoS Comput Biol. 8:e1002634.

11. Jirsa VK, Stacey WC, Quilichini PP, Ivanov AI, Bernard C (2014): On the nature of seizure dynamics. Brain. 137:2210-2230.

12. Naze S, Bernard C, Jirsa V (2015): Computational modeling of seizure dynamics using coupled neuronal networks: factors shaping epileptiform activity. PLoS Comput Biol. 11:e1004209.

13. Robinson PA, Rennie CJ, Rowe DL (2002): Dynamics of large-scale brain activity in normal arousal states and epileptic seizures. Physical Review E. 65:041924.

14. Wendling F, Bartolomei F, Bellanger JJ, Chauvel P (2002): Epileptic fast activity can be explained by a model of impaired GABAergic dendritic inhibition. Eur J Neurosci. 15:14991508.

15. Scheffer M, Bascompte J, Brock WA, Brovkin V, Carpenter SR, Dakos V, et al. (2009): Early-warning signals for critical transitions. Nature. 461:53-59.

16. Cerf R, Kahane P (2004): Criticality and synchrony of fluctuations in rhythmical brain activity: pretransitional effects in epileptic patients. Biol Cybern. 90:239-255.

17. Litt B, Esteller R, Echauz J, D'Alessandro M, Shor R, Henry T, et al. (2001): Epileptic seizures may begin hours in advance of clinical onset: a report of five patients. Neuron. 30:5164. 
18. McSharry PE, Smith LA, Tarassenko L (2003): Prediction of epileptic seizures: are nonlinear methods relevant? Nat Med. 9:241-242.

19. Mormann F, Andrzejak RG, Elger CE, Lehnertz K (2007): Seizure prediction: the long and winding road. Brain. 130:314-333.

20. Freestone DR, Karoly PJ, Peterson AD, Kuhlmann L, Lai A, Goodarzy F, et al. (2015): Seizure prediction: science fiction or soon to become reality? Curr Neurol Neurosci Rep. 15:1-9.

21. Lopes da Silva FH, Blanes W, Kalitzin SN, Parra J, Suffczynski P, Velis DN (2003): Dynamical diseases of brain systems: different routes to epileptic seizures. Biomedical Engineering, IEEE Transactions on. 50:540-548.

22. Leblois A, Boraud T, Meissner W, Bergman H, Hansel D (2006): Competition between feedback loops underlies normal and pathological dynamics in the basal ganglia. $J$ Neurosci. 26:3567-3583.

23. Van Albada SJ, Gray RT, Drysdale PM, Robinson PA (2009): Mean-field modeling of the basal ganglia-thalamocortical system. II: Dynamics of parkinsonian oscillations. J Theor Biol. 257:664-688.

24. Frank MJ, Loughry B, O'Reilly RC (2001): Interactions between frontal cortex and basal ganglia in working memory: a computational model. Cognitive, Affective, \& Behavioral Neuroscience. 1:137-160.

25. Frank MJ (2005): Dynamic dopamine modulation in the basal ganglia: a neurocomputational account of cognitive deficits in medicated and nonmedicated Parkinsonism. Cognitive Neuroscience, Journal of. 17:51-72.

26. Frank MJ, Seeberger LC, O'reilly RC (2004): By carrot or by stick: cognitive reinforcement learning in parkinsonism. Science. 306:1940-1943.

27. Rubin JE, Terman D (2004): High frequency stimulation of the subthalamic nucleus eliminates pathological thalamic rhythmicity in a computational model. J Comput Neurosci. 16:211-235.

28. Meijer HGE, Krupa M, Cagnan H, Lourens MAJ, Heida T, Martens HCF, et al. (2011): From Parkinsonian thalamic activity to restoring thalamic relay using deep brain stimulation: new insights from computational modeling. Journal of Neural Engineering. 8:066005.

29. Schiff SJ (2010): Towards model-based control of Parkinson's disease. Philosophical Transactions of the Royal Society of London A: Mathematical, Physical and Engineering Sciences. 368:2269-2308.

30. Popovych OV, Tass PA (2014): Control of abnormal synchronization in neurological disorders. Front Neurol. 5:268.

31. Feng X-J, Shea-Brown E, Greenwald B, Kosut R, Rabitz H (2007): Optimal deep brain stimulation of the subthalamic nucleus - a computational study. J Comput Neurosci. 23:265-282.

32. Tass PA, Silchenko AN, Hauptmann C, Barnikol UB, Speckmann E-J (2009): Longlasting desynchronization in rat hippocampal slice induced by coordinated reset stimulation. Physical Review E. 80:011902.

33. Wang J, Nebeck S, Muralidharan A, Johnson MD, Vitek JL, Baker KB (2016): Coordinated Reset Deep Brain Stimulation of Subthalamic Nucleus Produces Long-Lasting, Dose-Dependent Motor Improvements in the 1-Methyl-4-phenyl-1, 2, 3, 6-tetrahydropyridine Non-Human Primate Model of Parkinsonism. Brain Stimulation.

34. Rosin B, Slovik M, Mitelman R, Rivlin-Etzion M, Haber SN, Israel Z, et al. (2011): Closed-loop deep brain stimulation is superior in ameliorating parkinsonism. Neuron. 72:370384. 
35. Little S, Pogosyan A, Neal S, Zavala B, Zrinzo L, Hariz M, et al. (2013): Adaptive deep brain stimulation in advanced Parkinson disease. Ann Neurol. 74:449-457.

36. Tass PA (2007): Phase resetting in medicine and biology: stochastic modelling and data analysis. Berlin: Springer.

37. Tass PA (2001): Desynchronizing double-pulse phase resetting and application to deep brain stimulation. Biol Cybern. 85:343-354.

38. Tass PA, Klosterkötter J, Schneider F, Lenartz D, Koulousakis A, Sturm V (2003): Obsessive-compulsive disorder: development of demand-controlled deep brain stimulation with methods from stochastic phase resetting. Neuropsychopharmacology. 28:S27-34.

39. Tass PA, Popovych OV (2012): Unlearning tinnitus-related cerebral synchrony with acoustic coordinated reset stimulation: theoretical concept and modelling. Biol Cybern. 106:2736.

40. Niedermeyer E, Sherman DL, Geocadin RJ, Hansen HC, Hanley DF (1999): The burstsuppression electroencephalogram. Clinical EEG (electroencephalography). 30:99.

41. Roberts JA, Iyer KK, Finnigan S, Vanhatalo S, Breakspear M (2014): Scale-Free Bursting in Human Cortex following Hypoxia at Birth. J Neurosci. 34:6557-6572.

42. Sethna JP, Dahmen KA, Myers CR (2001): Crackling noise. Nature. 410:242-250.

43. Iyer KK, Roberts JA, Metsäranta M, Finnigan S, Breakspear M, Vanhatalo S (2014): Novel features of early burst suppression predict outcome after birth asphyxia. Annals of Clinical and Translational Neurology. 1:209-214.

44. Iyer KK, Roberts JA, Hellström-Westas L, Wikström S, Hansen-Pupp I, Ley D, et al. (2015): Early detection of preterm intraventricular hemorrhage from clinical electroencephalography. Crit Care Med. 43:2219-2227.

45. Iyer KK, Roberts JA, Hellström-Westas L, Wikström S, Hansen-Pupp I, Ley D, et al. (2015): Cortical burst dynamics predict clinical outcome early in extremely preterm infants. Brain. 138:2206-2218.

46. Ching S, Purdon PL, Vijayan S, Kopell NJ, Brown EN (2012): A neurophysiologicalmetabolic model for burst suppression. Proc Natl Acad Sci U S A. 109:3095-3100.

47. Roberts JA, Iyer KK, Vanhatalo S, Breakspear M (2014): Critical role for resource constraints in neural models. Front Syst Neurosci. 8:154.

48. Steyn-Ross ML, Steyn-Ross DA, Sleigh JW, Liley DTJ (1999): Theoretical electroencephalogram stationary spectrum for a white-noise-driven cortex: Evidence for a general anesthetic-induced phase transition. Physical Review E. 60:7299.

49. Bojak I, Liley DTJ (2005): Modeling the effects of anesthesia on the electroencephalogram. Physical Review E. 71:041902.

50. Hindriks R, van Putten MJAM (2012): Meanfield modeling of propofol-induced changes in spontaneous EEG rhythms. Neuroimage. 60:2323-2334.

51. Boly M, Moran R, Murphy M, Boveroux P, Bruno M-A, Noirhomme Q, et al. (2012): Connectivity changes underlying spectral EEG changes during propofol-induced loss of consciousness. J Neurosci. 32:7082-7090.

52. Kuhlmann L, Freestone DR, Manton JH, Heyse B, Vereecke HE, Lipping T, et al. (2016): Neural mass model-based tracking of anesthetic brain states. Neuroimage. (in press).

53. Bonsall MB, Wallace-Hadrill SMA, Geddes JR, Goodwin GM, Holmes EA (2012): Nonlinear time-series approaches in characterizing mood stability and mood instability in bipolar disorder. Proceedings of the Royal Society of London B: Biological Sciences. 279:916-924. 
54. Moore PJ, Little MA, McSharry PE, Goodwin GM, Geddes JR (2014): Mood dynamics in bipolar disorder. International Journal of Bipolar Disorders. 2:11.

55. Bonsall MB, Geddes JR, Goodwin GM, Holmes EA (2015): Bipolar disorder dynamics: affective instabilities, relaxation oscillations and noise. Journal of The Royal Society Interface. 12:20150670.

56. Daugherty D, Roque-Urrea T, Urrea-Roque J, Troyer J, Wirkus S, Porter MA (2009): Mathematical models of bipolar disorder. Communications in Nonlinear Science and Numerical Simulation. 14:2897-2908.

57. Steinacher A, Wright KA (2013): Relating the bipolar spectrum to dysregulation of behavioural activation: a perspective from dynamical modelling. PLoS One. 8:e63345.

58. Goldbeter A (2013): Origin of cyclicity in bipolar disorders: a computational approach. Pharmacopsychiatry. 46:S44-52.

59. Frank TD (2013): A limit cycle oscillator model for cycling mood variations of bipolar disorder patients derived from cellular biochemical reaction equations. Communications in Nonlinear Science and Numerical Simulation. 18:2107-2119.

60. Holmes EA, Bonsall MB, Hales SA, Mitchell H, Renner F, Blackwell SE, et al. (2016): Applications of time-series analysis to mood fluctuations in bipolar disorder to promote treatment innovation: a case series. Translational Psychiatry. 6:e720.

61. Critchley HD (2005): Neural mechanisms of autonomic, affective, and cognitive integration. J Comp Neurol. 493:154-166.

62. Critchley HD, Wiens S, Rotshtein P, Öhman A, Dolan RJ (2004): Neural systems supporting interoceptive awareness. Nat Neurosci. 7:189-195.

63. Paulus MP, Stein MB (2006): An insular view of anxiety. Biol Psychiatry. 60:383-387.

64. Huys QJM, Maia TV, Frank MJ (2016): Computational psychiatry as a bridge from neuroscience to clinical applications. Nat Neurosci. 19:404-413.

65. van de Leemput IA, Wichers M, Cramer AOJ, Borsboom D, Tuerlinckx F, Kuppens P, et al. (2014): Critical slowing down as early warning for the onset and termination of depression. Proc Natl Acad Sci U S A. 111:87-92.

66. Hyett MP, Breakspear MJ, Friston KJ, Guo CC, Parker GB (2015): Disrupted effective connectivity of cortical systems supporting attention and interoception in melancholia. JAMA Psychiatry. 72:350-358.

67. Hyett MP, Parker GB, Guo CC, Zalesky A, Nguyen VT, Yuen T, et al. (2015): Scene unseen: Disrupted neuronal adaptation in melancholia during emotional film viewing. NeuroImage: Clinical. 9:660-667.

68. Parker G, Hadzi-Pavlovic D, Wilhelm K, Hickie I, Brodaty H, Boyce P, et al. (1994): Defining melancholia: properties of a refined sign-based measure. The British Journal of Psychiatry. 164:316-326.

69. Friston KJ, Li B, Daunizeau J, Stephan KE (2011): Network discovery with DCM. Neuroimage. 56:1202-1221.

70. Phillips AJK, Robinson PA (2007): A quantitative model of sleep-wake dynamics based on the physiology of the brainstem ascending arousal system. J Biol Rhythms. 22:167-179.

71. Fulcher BD, Phillips AJK, Postnova S, Robinson PA (2014): A physiologically based model of orexinergic stabilization of sleep and wake. PLoS One. 9:e91982.

72. Yang D-P, McKenzie-Sell L, Karanjai A, Robinson PA (2016): Wake-sleep transition as a noisy bifurcation. Physical Review E. 94:022412. 
73. Robinson PA, Postnova S, Abeysuriya RG, Kim JW, Roberts JA, McKenzie-Sell L, et al. (2015): A Multiscale "Working Brain" Model. In: Bhattacharya BS, Chowdhury FN, editors. Validating Neuro-Computational Models of Neurological and Psychiatric Disorders: Springer International Publishing, pp 107-140.

74. Abeysuriya RG, Rennie CJ, Robinson PA (2015): Physiologically based arousal state estimation and dynamics. J Neurosci Methods. 253:55-69.

75. Abeysuriya RG, Robinson PA (2016): Real-time automated EEG tracking of brain states using neural field theory. J Neurosci Methods. 258:28-45.

76. Deco G, Rolls ET (2003): Attention and working memory: a dynamical model of neuronal activity in the prefrontal cortex. Eur J Neurosci. 18:2374-2390.

77. Deco G, Rolls ET, Romo R (2009): Stochastic dynamics as a principle of brain function. Prog Neurobiol. 88:1-16.

78. Deco G, Rolls ET (2004): A neurodynamical cortical model of visual attention and invariant object recognition. Vision Res. 44:621-642.

79. Wang X-J (2001): Synaptic reverberation underlying mnemonic persistent activity. Trends Neurosci. 24:455-463.

80. Compte A, Brunel N, Goldman-Rakic PS, Wang X-J (2000): Synaptic mechanisms and network dynamics underlying spatial working memory in a cortical network model. Cereb Cortex. 10:910-923.

81. Wang X-J (1999): Synaptic basis of cortical persistent activity: the importance of NMDA receptors to working memory. J Neurosci. 19:9587-9603.

82. Loh M, Rolls ET, Deco G (2007): A dynamical systems hypothesis of schizophrenia. PLoS Comput Biol. 3:e228.

83. Durstewitz D, Seamans JK (2008): The dual-state theory of prefrontal cortex dopamine function with relevance to catechol-o-methyltransferase genotypes and schizophrenia. Biol Psychiatry. 64:739-749.

84. Durstewitz D, Seamans JK, Sejnowski TJ (2000): Dopamine-mediated stabilization of delay-period activity in a network model of prefrontal cortex. J Neurophysiol. 83:1733-1750.

85. Rolls ET, Loh M, Deco G, Winterer G (2008): Computational models of schizophrenia and dopamine modulation in the prefrontal cortex. Nature Reviews Neuroscience. 9:696-709.

86. Yang GJ, Murray JD, Repovs G, Cole MW, Savic A, Glasser MF, et al. (2014): Altered global brain signal in schizophrenia. Proc Natl Acad Sci U S A. 111:7438-7443.

87. Anticevic A, Cole MW, Repovš G, Savic A, Driesen NR, Yang G, et al. (2013): Connectivity, Pharmacology, and Computation: Toward a Mechanistic Understanding of Neural System Dysfunction in Schizophrenia. Frontiers in Psychiatry. 4:169.

88. Anticevic A, Murray JD, Barch DM (2015): Bridging levels of understanding in schizophrenia through computational modeling. Clinical Psychological Science. 3:433-459.

89. Ratcliff R, Smith PL, Brown SD, McKoon G (2016): Diffusion Decision Model: Current Issues and History. Trends in Cognitive Sciences. 20:260-281.

90. Bogacz R, Brown E, Moehlis J, Holmes P, Cohen JD (2006): The physics of optimal decision making: a formal analysis of models of performance in two-alternative forced-choice tasks. Psychol Rev. 113:700.

91. Moustafa AA, Kéri S, Somlai Z, Balsdon T, Frydecka D, Misiak B, et al. (2015): Drift diffusion model of reward and punishment learning in schizophrenia: Modeling and experimental data. Behav Brain Res. 291:147-154. 
92. Mulder MJ, Bos D, Weusten JM, van Belle J, van Dijk SC, Simen P, et al. (2010): Basic impairments in regulating the speed-accuracy tradeoff predict symptoms of attentiondeficit/hyperactivity disorder. Biol Psychiatry. 68:1114-1119.

93. Banca P, Vestergaard MD, Rankov V, Baek K, Mitchell S, Lapa T, et al. (2015): Evidence accumulation in obsessive-compulsive disorder: the role of uncertainty and monetary reward on perceptual decision-making thresholds. Neuropsychopharmacology. 40:1192-1202.

94. Amit DJ, Brunel N (1997): Model of global spontaneous activity and local structured activity during delay periods in the cerebral cortex. Cereb Cortex. 7:237-252.

95. Brunel N, Wang X-J (2001): Effects of neuromodulation in a cortical network model of object working memory dominated by recurrent inhibition. J Comput Neurosci. 11:63-85.

96. Deco G, Rolls ET, Albantakis L, Romo R (2013): Brain mechanisms for perceptual and reward-related decision-making. Prog Neurobiol. 103:194-213.

97. Wang X-J (2002): Probabilistic decision making by slow reverberation in cortical circuits. Neuron. 36:955-968.

98. Garrett DD, Kovacevic N, McIntosh AR, Grady CL (2011): The importance of being variable. J Neurosci. 31:4496-4503.

99. McIntosh AR, Kovacevic N, Lippe S, Garrett D, Grady C, Jirsa V (2010): The development of a noisy brain. Arch Ital Biol. 148:323-337.

100. McIntosh AR, Kovacevic N, Itier RJ (2008): Increased brain signal variability accompanies lower behavioral variability in development. PLoS Comput Biol. 4:e1000106.

101. McIntosh AR, Vakorin V, Kovacevic N, Wang H, Diaconescu A, Protzner AB (2013): Spatiotemporal dependency of age-related changes in brain signal variability. Cereb Cortex. 24:1806-1817.

102. Beharelle AR, Kovačević N, McIntosh AR, Levine B (2012): Brain signal variability relates to stability of behavior after recovery from diffuse brain injury. Neuroimage. 60:15281537.

103. Mišić B, Mills T, Taylor MJ, McIntosh AR (2010): Brain noise is task dependent and region specific. J Neurophysiol. 104:2667-2676.

104. Garrett DD, Kovacevic N, McIntosh AR, Grady CL (2010): Blood oxygen leveldependent signal variability is more than just noise. J Neurosci. 30:4914-4921.

105. Garrett DD, Samanez-Larkin GR, MacDonald SW, Lindenberger U, McIntosh AR, Grady CL (2013): Moment-to-moment brain signal variability: a next frontier in human brain mapping? Neurosci Biobehav Rev. 37:610-624.

106. Maia TV, McClelland JL (2012): A neurocomputational approach to obsessivecompulsive disorder. Trends in Cognitive Sciences. 16:14-15.

107. Rolls ET, Loh M, Deco G (2008): An attractor hypothesis of obsessive-compulsive disorder. Eur J Neurosci. 28:782-793.

108. Verduzco-Flores S, Ermentrout B, Bodner M (2012): Modeling neuropathologies as disruption of normal sequence generation in working memory networks. Neural Networks. 27:21-31.

109. Maia TV, Cano-Colino M (2015): The role of serotonin in orbitofrontal function and obsessive-compulsive disorder. Clinical Psychological Science. 3:460-482.

110. Heinzle J, Aponte EA, Stephan KE (2016): Computational models of eye movements and their application to schizophrenia. Current Opinion in Behavioral Sciences. 11:21-29.

111. Cutsuridis V, Kumari V, Ettinger U (2014): Antisaccade performance in schizophrenia: a neural model of decision making in the superior colliculus. Front Neurosci. 8:13. 
112. Adams RA, Perrinet LU, Friston K (2012): Smooth pursuit and visual occlusion: active inference and oculomotor control in schizophrenia. PLoS One. 7:e47502.

113. Friston KJ, Daunizeau J, Kilner J, Kiebel SJ (2010): Action and behavior: a free-energy formulation. Biol Cybern. 102:227-260.

114. Adams RA, Aponte E, Marshall L, Friston KJ (2015): Active inference and oculomotor pursuit: The dynamic causal modelling of eye movements. J Neurosci Methods. 242:1-14.

115. Adams RA, Bauer M, Pinotsis D, Friston KJ (2016): Dynamic causal modelling of eye movements during pursuit: confirming precision-encoding in V1 using MEG. Neuroimage. 132:175-189.

116. Benson PJ, Beedie SA, Shephard E, Giegling I, Rujescu D, St. Clair D (2012): Simple viewing tests can detect eye movement abnormalities that distinguish schizophrenia cases from controls with exceptional accuracy. Biol Psychiatry. 72:716-724.

117. Engbert R, Mergenthaler K, Sinn P, Pikovsky A (2011): An integrated model of fixational eye movements and microsaccades. Proc Natl Acad Sci U S A. 108:E765-E770.

118. Roberts JA, Wallis G, Breakspear M (2013): Fixational eye movements during viewing of dynamic natural scenes. Front Psychol. 4:797.

119. Seghier ML, Friston KJ (2012): Network discovery with large DCMs. Neuroimage.

120. Rinzel J (1987): A formal classification of bursting mechanisms in excitable systems. In:

Teramoto E, Yamaguti M, editors. Mathematical topics in population biology, morphogenesis and neurosciences. New York: Springer, pp 267-281.

121. Izhikevich EM (2000): Neural excitability, spiking and bursting. International Journal of Bifurcation and Chaos. 10:1171-1266.

122. Mathys CD, Lomakina EI, Daunizeau J, Iglesias S, Brodersen KH, Friston KJ, et al. (2014): Uncertainty in perception and the Hierarchical Gaussian Filter. Front Hum Neurosci. $8: 825$.

123. Breakspear M, Stam CJ (2005): Dynamics of a neural system with a multiscale architecture. Philosophical Transactions of the Royal Society B: Biological Sciences. 360:10511074.

124. Stephan KE, Iglesias S, Heinzle J, Diaconescu AO (2015): Translational perspectives for computational neuroimaging. Neuron. 87:716-732.

125. Brodersen KH, Schofield TM, Leff AP, Ong CS, Lomakina EI, Buhmann JM, et al. (2011): Generative embedding for model-based classification of fMRI data. PLoS Comput Biol. 7:e1002079.

126. Eaton NR (2015): Latent variable and network models of comorbidity: toward an empirically derived nosology. Soc Psychiatry Psychiatr Epidemiol. 50:845-849.

127. Stephan KE, Bach DR, Fletcher PC, Flint J, Frank MJ, Friston KJ, et al. (2016): Charting the landscape of priority problems in psychiatry, part 1: classification and diagnosis. The Lancet Psychiatry. 3:77-83.

128. Frank MJ, Samanta J, Moustafa AA, Sherman SJ (2007): Hold your horses: impulsivity, deep brain stimulation, and medication in parkinsonism. Science. 318:1309-1312.

129. Huys QJM, Daw ND, Dayan P (2015): Depression: a decision-theoretic analysis. Annu Rev Neurosci. 38:1-23.

130. Huys QJM, Pizzagalli DA, Bogdan R, Dayan P (2013): Mapping anhedonia onto reinforcement learning: a behavioural meta-analysis. Biology of Mood \& Anxiety Disorders. 3:1.

131. Glimcher PW, Fehr E (2014): Neuroeconomics: Decision making and the brain. 2nd ed. San Diego: Academic Press. 


\section{Figure captions}

Figure 1: (duplicated from the companion primer): Noise-induced transitions in a simple bistable model (the Hopf-bifurcation model used in Ref. (10); similar ideas have been explored in biophysical seizure models $(4,6,7)$ ). See Appendix A of Part I (1) for further details of the model and definitions of technical terms. A: Bifurcation diagram for a supercritical Hopf bifurcation. For $\beta<0$, the only attractor is a stable fixed point ( $r=0$, black solid line). For $\beta>$ 0 , the fixed point is unstable (black dotted line), and a stable limit cycle (green) emerges with radius $r>0$. The green line depicts the radius of the limit cycle for the corresponding value of $\beta$. B: Bifurcation diagram for a subcritical Hopf bifurcation and bistability. The $r=0$ fixed point behaves the same as in panel A. The difference here is that the limit cycle that emerges at $\beta=0$ exists for $\beta<0$ and is unstable (red). This unstable limit cycle then meets a stable limit cycle (green) at a "saddle-node" bifurcation of periodic orbits. There is thus a region of bistability where the fixed point (black) and the upper limit cycle (green) are both stable, separated by an unstable limit cycle (red). C-F: Left column: time series for one of the two Cartesian coordinates in the phase space. Right column: trajectories in phase space (blue) with stable attractor (green) and unstable "separatrix" (red) that demarcates the boundary across which a sudden transition (e.g., seizure onset) occurs. The polar radial coordinate $r$ in panels A and B is given by $r^{2}=x^{2}+y^{2}$. C,D: The low-amplitude case: the trajectory remains well within the transition boundary, exhibiting only low-amplitude fluctuations (e.g., healthy non-seizure dynamics). E,F: The high-amplitude case: random fluctuations cross the boundary triggering high-amplitude oscillations (e.g., a seizure).

Figure 2: Stochastic neural modeling of neuronal activity in melancholia $(66,67)$. A: Spatial independents components analysis was used to identify a constellation of canonical spatial modes, namely auditory (AUD), default mode (DMN), midline prefrontal "executive" (EXC), left and right insulae (INS), left and right fronto-parietal attention (LFP, RFP), and the medial visual pole (MVP). Stochastic DCM was then used to infer interactions between these modes; i.e., the non-zero elements of the coupling matrix A from resting state $(66,67)$ and during passive movie viewing $(66,67)$. B: Methodological schema, showing extraction of the time 
series from each node (ICA component) followed by model inversion to yield subject-specific hidden time series. In most nodes (especially visual and auditory but also insula), hidden states in different subjects watching the same movie exhibit inter-subject correlations (due to attention to the same stochastic stimulus stream). Adapted from Ref. (67) with permission.

Figure 3: Narcolepsy in a stochastic model of the ascending arousal system with bistability between sleep and wake. Periods of sleep (black) and wake (white) are plotted as a function of orexin levels (a neuropeptide, parameterized by $v_{m x}$ ) across two-day model simulations. Adapted from Ref. (71) with permission. 